\title{
An Integrated Low-Noise Charge-Sensitive Preamplifier with Virtually Unlimited Spectroscopic Dynamic Range*
}

*Patent Pending

\author{
A. Pullia, F. Zocca and S. Capra
}

\begin{abstract}
A low-noise ASIC preamplifier for semiconductor detectors has been built and characterized, which is able to provide a linear spectroscopic measurement of the detector charge signals even when its output voltage is saturated. The preamplifier works in a conventional mode, i.e. as an active current integrator, when the output signal is below a preset threshold. If the signal is larger the preamplifier operates in a non-standard controlled fast-reset mode, which is effective in providing a linear measurement of the detector signal charge even when the preamplifier works close to or in saturation. The experimental relation between the measurement and the input charge keeps perfectly linear irrespective of the non-linearity of the preamplifier working regime, which yields a dynamic-range boost of more than one order of magnitude.
\end{abstract}

\section{INTRODUCTION}

NEXT generation nuclear-physics experiments to be 1 executed in international dedicated facilities, like GSI, LNL, and GANIL in Europe, will employ highly-segmented HPGe (High Purity Germanium) detectors for gamma-ray measurement and tracking [1]-[5], and will make use of radioactive ion beams to investigate the nuclear structure under very extreme condition of stability. Both the detectors and the front-end electronics will have to work in new and hostile conditions that could substantially spoil their spectroscopic performance. One of the most critical issues is related to the foreseen background of charged particles, like pions or kaons with energies up to $100 \mathrm{MeV}$ or more. When an energetic charged particle hits a detector crystal it gets fully absorbed and generates a huge charge signal that can easily put the front-end electronics in a prolonged saturation state, paralyzing the system and compromising its functionalities. So, it is of paramount importance that the front-end is capable to properly manage such large charge signals. The simplest way to address this issue is the use of discrete-component preamplifiers working on very high power supplies of $\pm 12 \mathrm{~V}$ or $\pm 24 \mathrm{~V}$ [6][14]. These preamplifiers can reach a huge energy range of $\sim 150 \mathrm{MeV}$ while keeping the typical noise performance required for gamma-ray spectroscopy. But discrete-component circuits are very bulky and consume some hundreds milliwatt

A. Pullia is with the Department of Physics, University of Milano, Italy, and INFN of Milano, Italy (email: alberto.pullia@mi.infn.it).

F. Zocca was with INFN of Milano, Italy, and is now with CERN, Switzerland

S. Capra is with the Physics Department of University of Milano, Italy per channel when using such large power supplies. For nextgeneration highly-segmented detectors the use of ASIC preamplifiers is going to become mandatory, because the channel density and the power budget requirements are getting incompatible with discrete-component solutions. So the question is: how to treat extra large charge signals with an integrated preamplifier?

The use of high power supplies is not viable with integrated circuits owing to the "scaling down" process, which yields a lower and lower breakdown voltage for the individual devices. As an example the maximum rating for power supplies is $\pm 2.5 \mathrm{~V}$ in a $0.35 \mu \mathrm{m}$ CMOS technologies widely used for lownoise analogue designs. Using so low power supplies the output voltage swing of an integrated charge-sensitive preamplifier for semiconductor detectors is typically about $\pm 1.5 \mathrm{~V}$ This translates into an energy range of $10 \mathrm{MeV}$ when using the typical sensitivity of $150 \mathrm{mV} / \mathrm{MeV}$ as needed to achieve energy resolutions of $0.1-0.2 \% @ 1-2 \mathrm{MeV}$ with a noise floor $<1 \mathrm{keV}$ fwhm. So there is no way to impede that an ASIC preamplifier enters a saturation state when the aforementioned energetic charged particles hit the detector, and the discussion should be mostly focused on how to usefully treat a saturated preamplifier. In this paper we discuss this carefully and introduce an innovative circuit technology for ASIC charge preamplifiers that yields

(i) a short recovery time from overloads caused by large signals. As an example a $100 \mathrm{MeV}$ signal is fully reset in $10 \mu \mathrm{s}$

(ii) the ability to perform a precise and linear measurement of the amplitude of the detector signals that cause preamplifier saturation

Using the proposed technology the measurement dynamic range of a single ASIC preamplifier may easily reach the unprecedented figure of $10^{5}$.

\section{TECHNIQUE}

In this Section we briefly describe the technique used in the integrated Charge-Sensitive Preamplifier (CSP) we have designed, realized and test bench characterized. It embodies a patented cutting-edge circuit technology [15] that makes highresolution amplitude measurements possible also in the case of detector signals so large to cause a soft or deep saturation of 


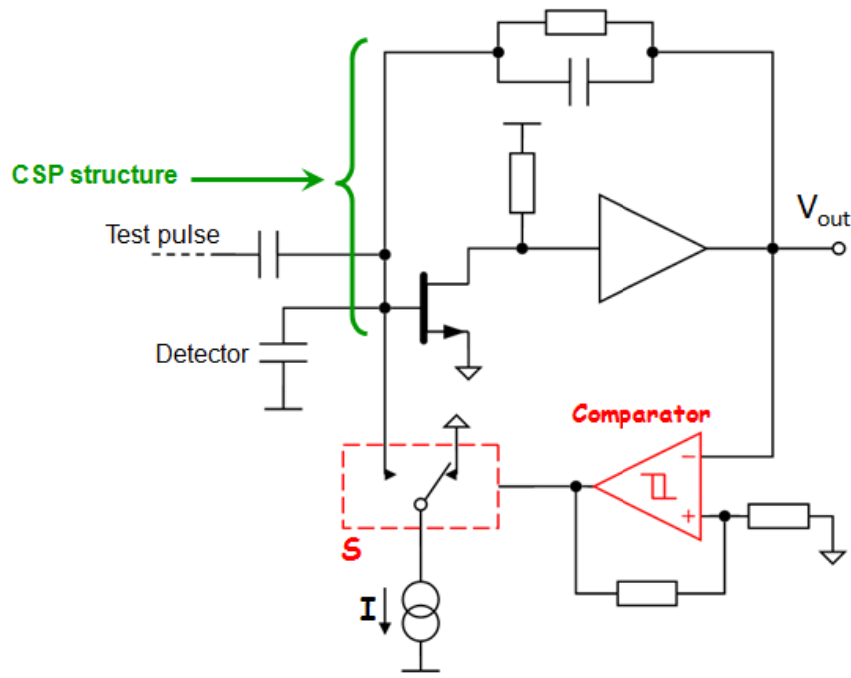

Fig.1. Simplified schematic diagram of the realized preamplifier. The controlled fast-reset feature is implemented through a Schmitt-trigger camparator, a constant current sink "I", and a double-throw CMOS switch "S".

the charge preamplifier. A simplified schematic diagram of the circuit is shown in Fig. 1.

The upper part of the circuit is a negative-feedback JFETCMOS CSP with continuous-time resistive reset [16]-[19], or, in other words, a low-noise active current integrator. The innovation consists of the three more devices shown in the lower part of the circuit: a Schmitt-trigger comparator, a precise constant current sink "I" and a double-throw CMOS switch "S" connected to the input node of the circuit. When the events are gamma rays of typical amplitude, i.e. a few $\mathrm{MeV}$ or a few hundreds millivolt as translated in terms of output voltage, the comparator stays in its low state, which keeps the switch in the right position. At this time current "I" is flown to ground and does not interfere with the CSP action of the circuit. But when a signal overcomes a preset threshold the comparator commutates to its high state, which drives the switch to the left position. At this time current "I" is forced to flow through the input node of the preamplifier, which begins a process of controlled removal of the physical charge provided by the detector. The process is controlled because driven by a precisely constant current "I". The larger is "I" the faster is the charge removal process. When the output voltage gets back to the zero volt baseline the comparator back commutates to its low state and the charge removal process terminates, which closes the controlled fast-reset phase. We set "I" in such a way that the reset time is $\sim 10 \mu$ s for a $100 \mathrm{MeV}$ energy release into the germanium detector. Notice that the larger is the signal charge delivered by the detector, the longer is the time required to fully remove it at a given removal rate. The key point is that reset time is strictly proportional to the charge by the very definition of electrical current. Current in fact is charge over time, and a constant current removes a precise amount charge per unit time. There is no way out of this and the linearity property is going to hold irrespective of

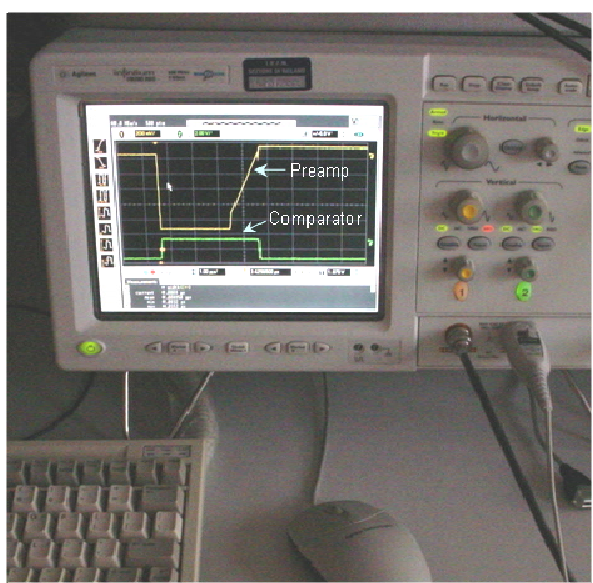

Fig. 2. Signals seen on the oscilloscope. The upper track is the preamplifier output signal in response to a large over-threshold test signal. The lower track is the corresponding comparator signal.

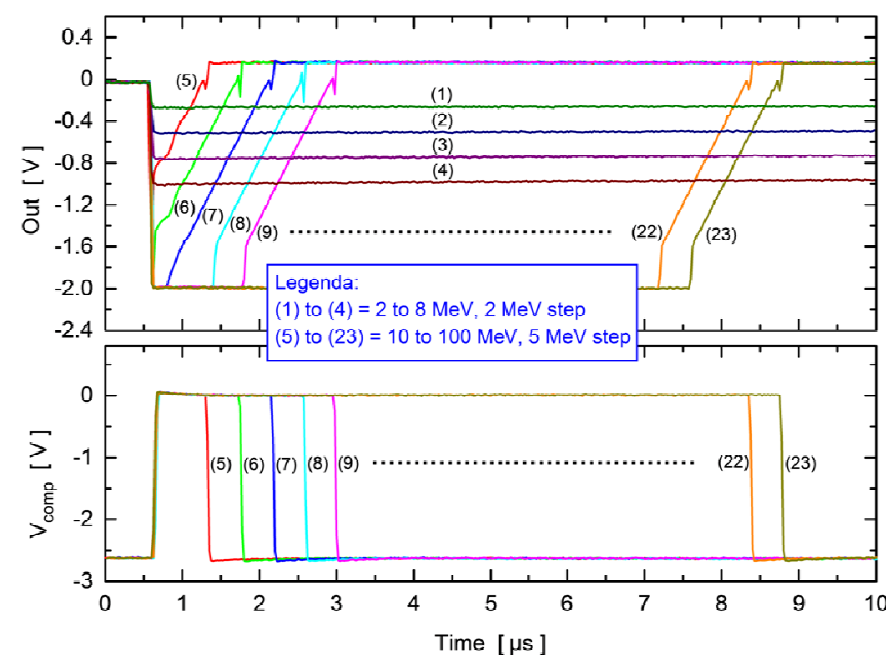

Fig. 3. Experimental response of the circuit to test pulses equivalent to events of 2 to $100 \mathrm{MeV}$ in germanium. On top and bottom the signals seen at the preamplifier and the comparator outputs are shown.

the working regime of the preamplifier, even if it is highly non-linear over most part of the reset process.

\section{CIRCUIT REALIZATION AND FIRST CHARACTERIZATION}

The circuit has been realized as a prototype in a low-noise $5 \mathrm{~V} 0.35 \mu \mathrm{m}$ CMOS technology, and characterized in the lab. The first signals which prove the functionality of the fast reset feature are shown in Fig. 2 as seen on the scope.

In Fig. 3 a set of such signals is shown for input signals of increasing amplitude. In the upper part of the figure the preamplifier output signals are shown. Signals from (1) to (4), or from 2 to $8 \mathrm{MeV}$, are under the comparator threshold and hence the circuit behaves like a conventional CSP providing step signals with a slow exponential decay in the ms time scale. Signals from (5) to (23), or from 10 to $100 \mathrm{MeV}$, are over threshold and hence the circuit executes a controlled fast reset of the signal charge. For these cases the relevant signals 


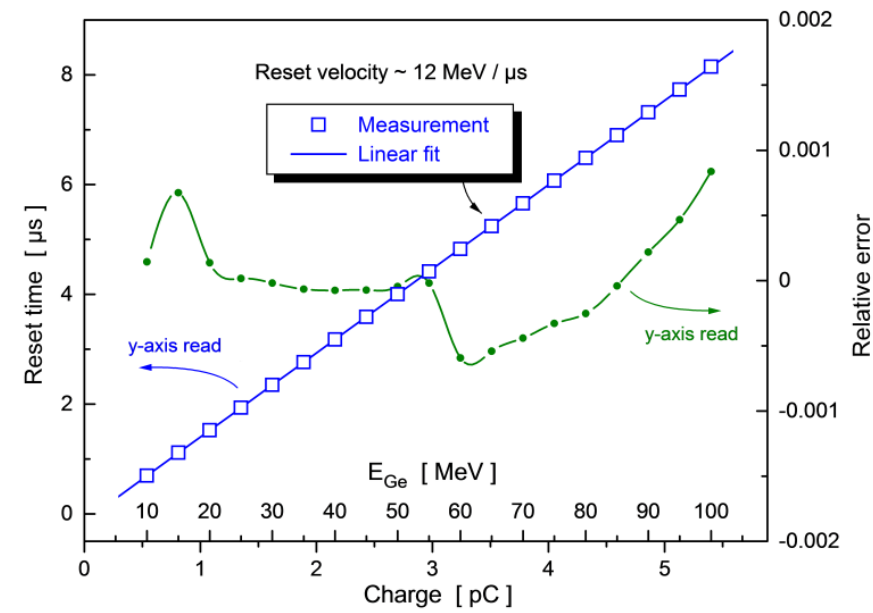

Fig. 4. Plot of the widths of the comparator signals, i.e. the reset times, as a function of the test pulse amplitudes in terms of injected charge or equivalent energy in germanium.

are those seen at the comparator output, shown in lower Fig. 3. As said the width of these signals, or the reset times, is expected to be strictly proportional to the signal charge itself. In Fig. 4 this property is shown where the experimental reset time is plot vs. the signal charge generated by the events. The $\mathrm{x}$-axis shows also the equivalent event energy considering the energy-to-charge conversion factor of germanium of $2.92 \mathrm{eV} /$ pair. The relation is indeed linear in the full range of available experimental data. Evaluating the deviation of the experimental data from the linear fitting a non linearity error is obtained below $\pm 0.1 \%$ over the full range. We have observed that the irregular behavior of the error at 15 and $55 \mathrm{MeV}$ is more related to the instrumentation used for the measurement than to the circuit itself. This proves experimentally the concept of controlled fast reset.

In Fig. 5 the response of the circuit is shown to a sequence of an extra large event of $40 \mathrm{MeV}$ and a much smaller event of 1 to $9 \mathrm{MeV}$ occurring only $4 \mu \mathrm{s}$ after. Typically the second event could not be observed ought to a prolonged saturation of the preamplifier lasting $1 \mathrm{~ms}$ or more. But thanks to the controlled fast-reset feature the large signal pulse lasts $3 \mu$ s only and the second event is cleanly revealed. This feature would be very useful in decay spectroscopy applications.

In Fig. 6 the room temperature Equivalent Noise Charge of the circuit is shown as a function of the shaping time for detector capacitances of 0 and $15 \mathrm{pF}$ (lower and upper curve). The fitting to the experimental data and the three disentangled typical noise components are also shown. [20]. The noise is $\sim 160$ r.m.s. electrons with a detector capacitance of $15 \mathrm{pF}$ at a shaping time of $6 \mu \mathrm{s}$, which is fully adequate for gamma spectroscopy. The noise is expected to diminish further when installing preamplifier in the detector cryostat and cooling it at cryogenic temperature. Another way to improve the noise would be use of a higher quality laminate for the PCB where the input transistor is mounted. In fact as shown in Fig. 6 the dominant noise contribution comes from the flat component (blue dashed line), related partly to the $1 / \mathrm{f}$ noise of the input

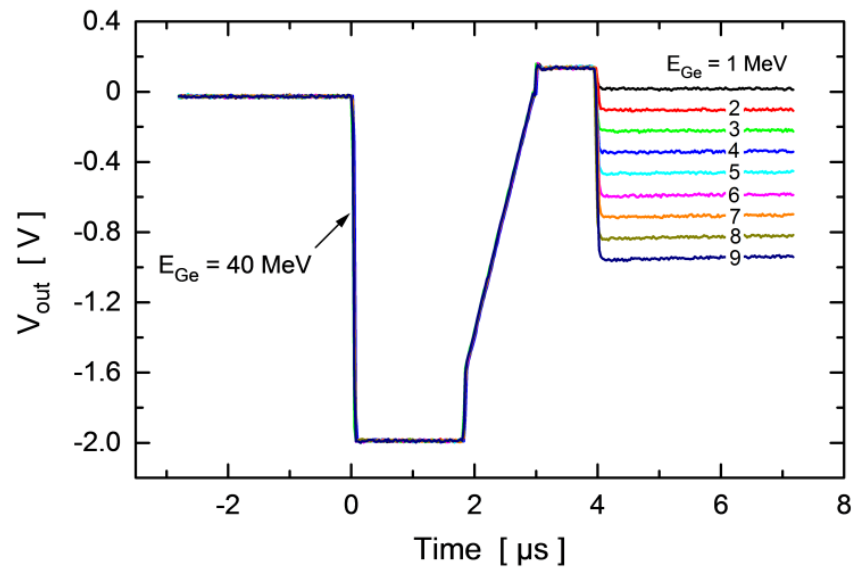

Fig. 5. Response of the preamplifier to a sequence of an extra large $(40 \mathrm{MeV})$ and a smaller signal (in the 1 to $9 \mathrm{MeV}$ range). The smaller signals.occur only $4 \mu$ s after the large one but are cleanly resolved.

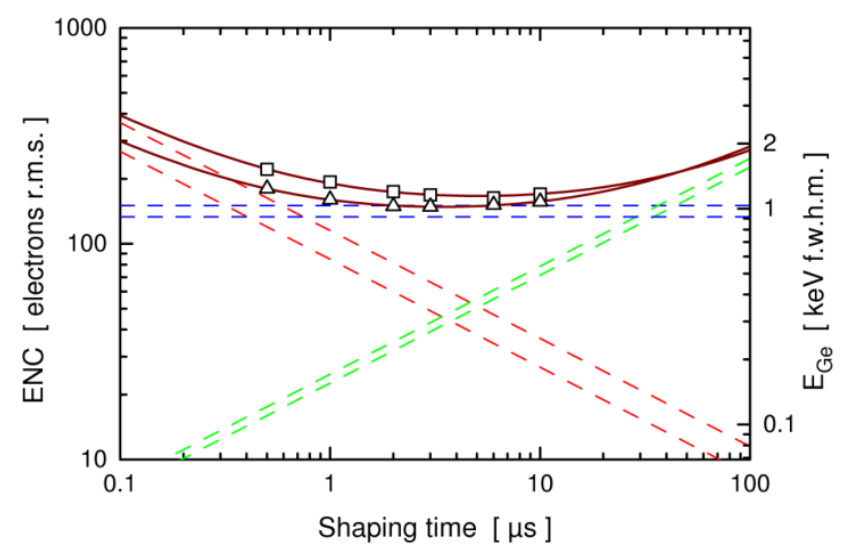

Fig. 6. Equivalent Noise Charge of the preamplifier vs. shaping time as taken at room temperature. The lower and upper curves are for detector capacitances of 0 and $15 \mathrm{pF}$. The fitting to the noise data and the three typical disentangled noise contributions are also shown.

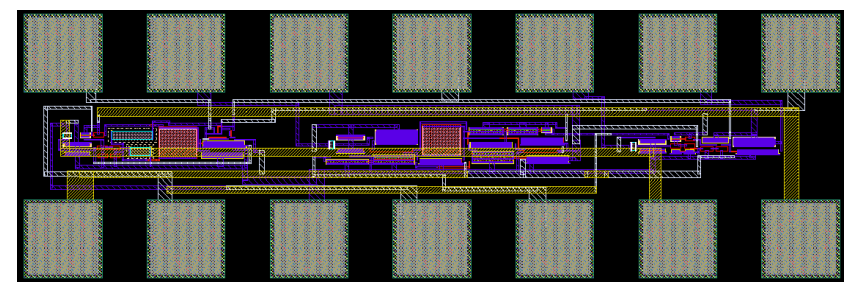

Fig. 7. Layout of the realized chip. The area occupancy is $700 \times 100 \mu \mathrm{m}^{2}$ excluding the bonding pads. The size of each bonding pad is $90 \times 90 \mu \mathrm{m}^{2}$

transistor but mostly to the dielectric noise of the insulators surrounding the input node of the preamplifier [21].

The layout of the tested circuit is shown in Fig. 7. The area occupancy is $700 \times 100 \mu \mathrm{m}^{2}$ excluding the bonding pads, and the power consumption is $\sim 30 \mathrm{~mW}$. 


\section{REFERENCES}

[1] AGATA homepage, URL: http://www-w2k.gsi.de/agata/

[2] S. Akkoyun et al., "AGATA-Advanced GAmmaTrackingArray", Nucl. Instrum. and Meth., vol. A 668, pp. 26-58, 2012

[3] GRETINA homepage, URL: http://grfs1.lbl.gov/

[4] A. Pullia et al., "Cross-Talk Limits of Highly Segmented Semiconductor Detectors", IEEE Trans. Nucl. Sci., vol. 58, no. 3, pp. 1201-1205, 2011

[5] A. Pullia et al., "Characterization of HPGe-Segmented Detectors from Noise Measurements", IEEE Trans. Nucl. Sci., vol. 51, no. 6, pp. 30863089, 2004

[6] G. Pascovici et al., "Low noise, dual gain preamplifier with built in spectroscopic pulser for highly segmented high-purity germanium detectors", WSEAS Trans. on Circuits and Systems, vol. 7, no. 6, pp. 470-481, 2008

[7] A. Pullia and F. Zocca, "A low-noise preamplifier for $\gamma$-ray sensors with add-on device for large-signal management", Nucl. Instrum. and Meth., vol. A545, pp. 784-792, 2005

[8] A. Pullia et al., "An Advanced Preamplifier for Highly Segmented Germanium Detectors", IEEE Trans. Nucl. Sci., vol. 53, no. 5, pp. 2869-2875, 2006

[9] F. Zocca et al., "A Smart Reset Circuit for Low-Noise Preamplifiers of X- $\gamma$ Ray Sensor Signals", IEEE Trans. Nucl. Sci., vol. 54, no. 1, pp. 197-201, 2007

[10] F. Zocca et al., "A Time-Over-Threshold Technique for Wide Dynamic Range Gamma-Ray Spectroscopy With the AGATA Detector", IEEE Trans. Nucl. Sci., vol. 56, no. 4, pp. 2384-2391, 2009

[11] A. Pullia et al., "A 'cold' discharge mechanism for low-noise fast charge amplifiers", IEEE Trans. Nucl. Sci., vol.48, no.3, pp. 530-534, 2001
[12] A. Pullia et al., "Extending the dynamic range of nuclear pulse spectrometers", Rev. Sci. Instrum., vol. 79, p. 036105-01, 2008, doi: $10.1063 / 1.2894305$

[13] F. Zocca et al., "Design and Optimization of Low-Noise WideBandwidth Charge Preamplifiers for High Purity Germanium Detectors", IEEE Trans. Nucl. Sci., vol. 55, no. 2, pp. 695-702, 2008

[14] A. Pullia et al, "Low-Noise Amplification of $\gamma$-Ray Detector Signals in Hostile Environments", IEEE Trans. Nucl. Sci., vol. 53, no.3, pp. 17441748,2006

[15] A.Pullia and F. Zocca, "Technique for measuring detector signals", Italian Patent n. MI2012A001560, 19 Sept 2012, URL: http://www.unimi.it/ricerca/brevetti/59851.htm

[16] A. Pullia et al, "Cryogenic Performance of a Low-Noise JFET-CMOS Preamplifier for HPGe Detectors", IEEE Trans. Nucl. Sci., vol. 57, no. 2, pp. 737-742, 2010

[17] A. Pullia et al., "A JFET-CMOS Fast Preamplifier for Segmented Germanium Detectors", IEEE Trans. Nucl. Sci., vol. 55, no. 1, pp. 591594, 2008

[18] A. Pullia et al., "A Compact VLSI dc Restorer for Multichannel X- $\gamma$ Ray Detectors", vol.. 52, no. 5, pp. 1643-1646, 2005

[19] C. Cattadori et al., "The GERmanium Detector Array read-out: Status and developments", Nucl. Instrum. and Meth., vol. A572, pp. 479-480, 2007

[20] G. Bertuccio and A. Pullia, "A method for the determination of the noise parameters in preamplifying systems for radiation detectors", Rev. Sci. Instrum., vol. 64, no. 11, pp. 3294-3298, 1993

[21] A. Pullia, G. Bertuccio, "Resolution limits of silicon detectors and electronics for soft X-ray spectroscopy at non cryogenic temperatures", Nucl. Instrum. and Meth., vol. A380, pp. 1-5, 1996 